\title{
Nanoscale
}

PAPER
Check for updates

Cite this: Nanoscale, 2019, 11, 5951

\section{Evolution of local conductance pathways in a single-molecule junction studied using the three-dimensional dynamic probe method $\uparrow$}

\author{
Atsushi Taninaka, Shoji Yoshida, Yoshihiro Sugita, Osamu Takeuchi and \\ Hidemi Shigekawa (D) *
}

\begin{abstract}
Understanding of the dynamics of the bonding states of molecules with electrodes while the molecular conformation is changed is particularly important for elucidating the details of electrochemical devices as well as molecular devices in which the reaction dynamics of the electrodes and molecules plays an important role, such as in fuel cells, catalysis and bioelectrochemical devices. However, it has been difficult to make measurements when the distance between counter electrodes is short, namely, the molecule is raised from a lying form, almost parallel and close to the electrodes, toward a standing form and vice versa. We previously have developed a method called the three-dimensional (3D) dynamic probe method, which enables conductance measurement while the conformation of a single-molecule junction is precisely controlled by scanning tunneling microscopy (STM) techniques. Here, by combining this method with density functional theory (DFT) calculations, it has become possible to simultaneously consider the effects of the dynamics of molecular structures and the bonding states at the electrodes on the local transmission pathways, local-bond contributions to conductance. Here, by performing an analysis on 1,4-benzenediamine (BDA) and 1,4-benzenedithiol (BDT) single molecule junctions, we have observed, for the first time, the effect of a change in the molecular conformations and bonding states on the local transmission pathways for a short Au electrode distance condition.
\end{abstract}

Received 23rd January 2019 Accepted 1st March 2019

DOI: 10.1039/c9nr00717b

rsc.li/nanoscale unknown and it is difficult to analyze whether the change in the measured conductance corresponds to the change in the conduction path due to the change in the contact structure or to the change in the transmittance due to the change in the molecular conformation.

Recently, a method that enables conductance measurement while changing the angle of the molecule to the substrate without destroying the junction has been developed as a type of BJ method; ${ }^{6,11,17}$ however, it is still difficult to analyze the structure of the bonding states. Since the dynamics of the contact point between the molecule and substrate affects the scattering of electrons when electrons are injected, it is necessary to simultaneously elucidate the conduction characteristics and the structures of the bonding states.

We previously developed a method called the three-dimensional (3D) dynamic probe method, which enables conductance measurement while the conformation of a single-molecule junction is precisely controlled by scanning tunneling microscopy (STM) techniques. ${ }^{18-21}$ By combining this method with density functional theory (DFT) calculations, it has become possible to simultaneously consider the effects of the dynamics of molecular structures and the bonding states at the electrodes on the local transmission pathways and conductance. $^{18-20,22-24}$
Faculty of Pure and Applied Sciences, University of Tsukuba, Tsukuba 305-8571, Japan.E-mail: hidemi@ims.tsukuba.ac.jp

$\dagger$ Electronic supplementary information (ESI) available. See DOI: 10.1039/ c9nr00717b 
However, the measurements in the previous studies were carried out with a gold STM tip sufficiently retracted from the substrate surface, namely molecules were in a standing form and thus formed junctions with the electrodes only at their two ends. When a molecule is in a lying form, almost parallel and close to the electrodes, the interaction between the molecule and the substrate is considered to be stronger, and conduction pathways are considered to exist between the electrodes via various parts of the molecule instead of a single flow through the molecule. As the molecule is raised, such direct conduction pathways between the electrodes begin to break and the bonding state changes into structures where junctions are formed only at the two ends of the molecule and an influence of the structure peculiar to each molecule appears. Although it is very important to understand the conductance properties appearing in such a dynamic change, as described above, they have not yet been investigated.

To clarify the change in the bonding states while the molecular conformation is varied from a lying to standing conformation and vice versa, we carried out 3D dynamic probe experiments and DFT simulations on 1,4-benzenediamine (BDA) and 1,4-benzenedithiol (BDT) molecules for comparison. BDA and BDT are compounds having one benzene ring. BDA is not connected to $\mathrm{Au}$ adatoms on the substrate but its lone pair forms a bond, which is expected to attract the molecule to the substrate and prevent the rotation of the molecule. ${ }^{19}$ In contrast, BDT, one of the simplest molecules which has often been used for BJ analysis, ${ }^{5,6,8-10,20}$ forms bonds with the Au adatoms on both electrodes, which allows rotational motion during a conformational change. ${ }^{20}$ Understanding of such differences is important for elucidating the details of electrochemical devices as well as molecular devices in which the reaction dynamics of the electrodes and molecules plays an important role, such as in fuel cells, catalytic actions and bioelectrochemical devices. ${ }^{1-4}$

\section{Experimental}

\section{Sample and tip preparation}

A clean flat $\mathrm{Au}(111)$ surface was prepared by evaporating $\mathrm{Au}$ of $100 \mathrm{~nm}$ thickness on a mica substrate that had been subjected to Ar sputtering (5 $\mathrm{min}, 1 \mu \mathrm{A} \mathrm{cm}{ }^{-2}$ ) and annealing (30 min, $700 \mathrm{~K})$ for 3-5 cycles. Then the $\mathrm{Au}(111)$ surface was exposed to BDA or BDT molecules introduced through a variable leak valve (10 s, partial pressure: $1.0 \times 10^{-6} \mathrm{~Pa}$ ). The STM tip was formed by cutting a Au wire ( $0.3 \mathrm{~mm}$ diameter). All measurements were carried out under vacuum $\left(<5.0 \times 10^{-8} \mathrm{~Pa}\right)$ using an Omicron LT STM system. By cooling the STM unit with liquid nitrogen for over one week, the temperature in the STM chamber was maintained at $83 \mathrm{~K}$ and the thermal drift was reduced.

\section{Experimental scheme and measurements}

Fig. 1a and b show the schematics of the molecular structures of BDA and BDT deposited on a $\mathrm{Au}(111)$ surface, respec-
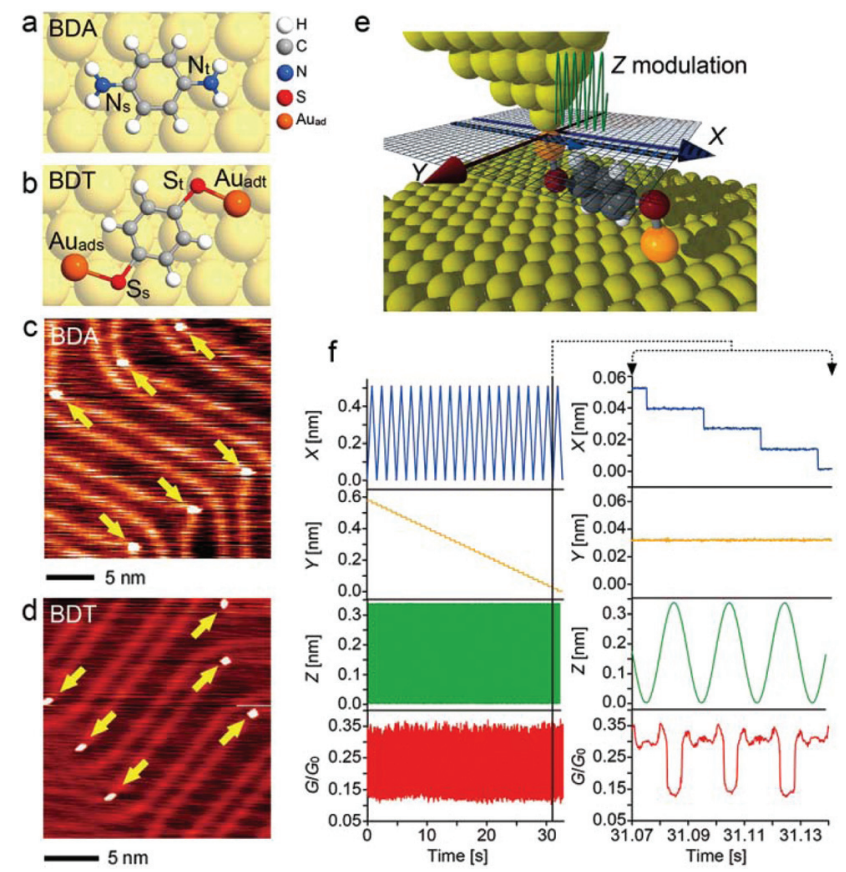

Fig. 1 Measurement setup. (a, b) Schematics of the molecular structures of BDA and BDT deposited on a $A u(111)$ surface, respectively. (c, d) STM images of BDA and BDT molecules on a Au (111) surface, respectively. (e) Schematic of the 3D dynamic probe method. (f) Schematic of the measurement scheme.

tively. ${ }^{25,26}$ In BDA, the $\mathrm{N}$ atoms connected to the surface side and the probe (STM tip) side are denoted by $\mathrm{N}_{\mathrm{S}}$ and $\mathrm{N}_{\mathrm{t}}$, respectively. On the other hand, BDT adsorbs with a $\mathrm{Au}$ adatom bound to thiol groups at both ends. ${ }^{20}$ For convenience, we denote the Au atoms on the substrate side and probe (tip) side as $\mathrm{Au}_{\mathrm{ads}}$ and $\mathrm{Au}_{\mathrm{adt}}$, respectively, and the $\mathrm{S}$ atoms bonded to $\mathrm{Au}_{\mathrm{ads}}$ and $\mathrm{Au}_{\mathrm{adt}}$ atoms are indicated by the slightly smaller red circles and named $S_{S}$ and $S_{t}$, respectively. Fig. 1c and $d$ show STM images obtained after the deposition of BDA and BDT molecules on a Au (111) surface, respectively. Similar to that in previous papers, ${ }^{19,20}$ it was confirmed that single BDT and BDA molecules were adsorbed at the elbow sites of the herringbone structure of the $\mathrm{Au}$ (111) surface, as shown by yellow arrows.

After observing a target BDA or BDT molecule $\left(V_{\mathrm{S}}=0.2 \mathrm{~V}\right.$, $I=+0.2 \mathrm{nA}$ ), the STM tip was moved above it. When the molecule was picked up, a rapid increase in conductance was observed. Since the molecular junction was unstable above the herringbone structure, we moved the junction away from the structure for measurement. Then the feedback was turned off and the sample bias voltage $V_{\mathrm{S}}$ was decreased to $10 \mathrm{mV}$ because the bond was unstable at high bias voltages. Then, the STM tip was moved back and forth in the Z-direction in accordance with a sinusoidal function $\left(z_{\mathrm{p}-\mathrm{p}}=0.224 \mathrm{~nm}\right.$, $50 \mathrm{~Hz}$ ), and was made to slowly approach the target molecule. The formation of a junction was confirmed by a rapid increase in current. After the formation was confirmed, the STM tip was moved back slightly $(0.14 \mathrm{~nm})$ to ensure a stable measure- 

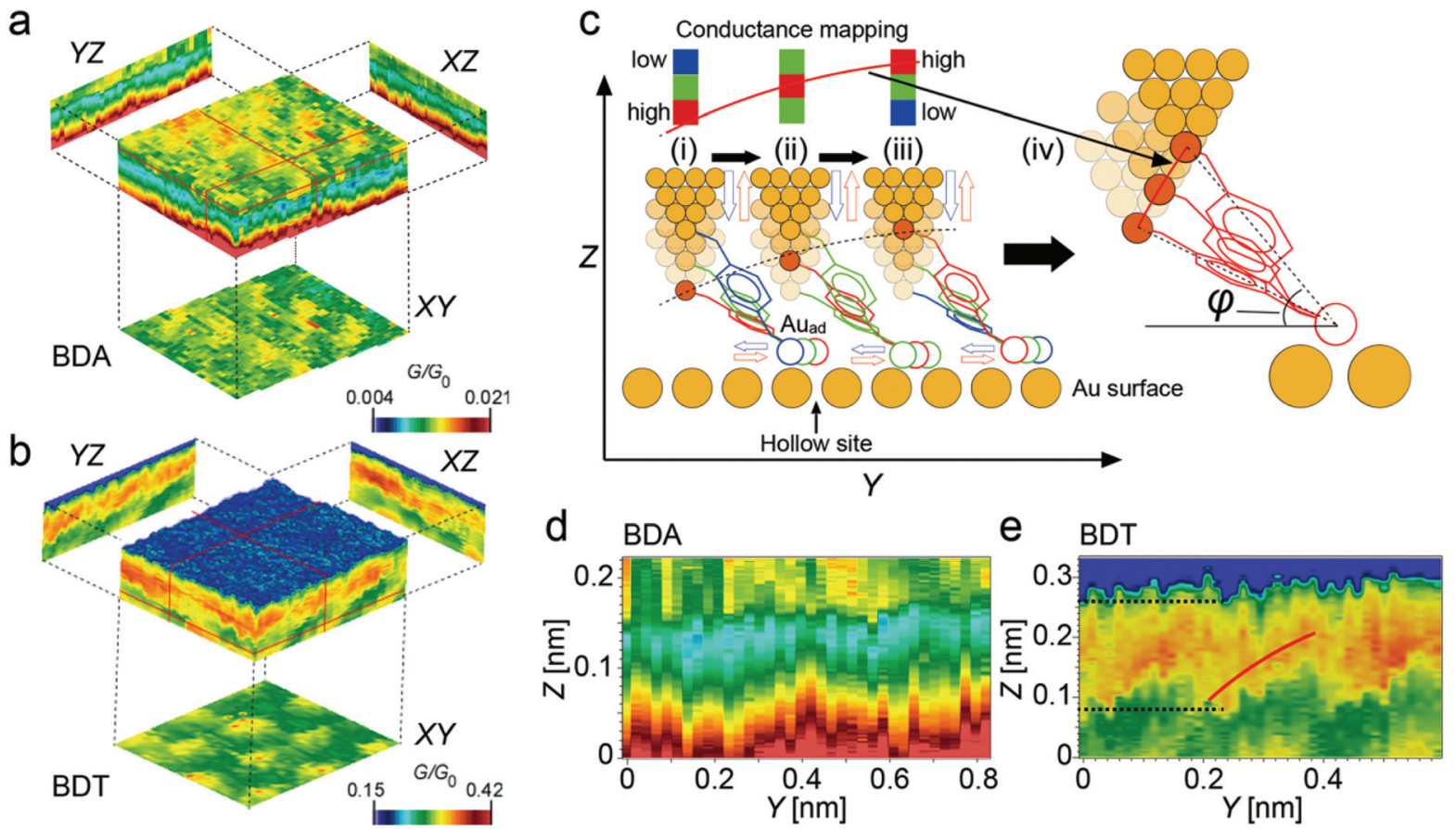

Fig. 2 3D plot and the analysis method. (a, b) 3D plots obtained for BDA and BDT molecules, respectively. (c) Schematic diagram to determine the adsorption angle. $\varphi$ represents the angle between the molecule and the substrate considering the direction of the molecule (see Fig. S1 $\uparrow$ for details). (d, e) $Y Z$ cross sections for the cases of BDA and BDT, respectively.

ment, and a two-dimensional scan was carried out step by step after each cycle of $Z$-modulation $(X, Y, Z: 56 \times 36 \times 200$ and $40 \times 40 \times 200$ points in the case of Fig. $2 a$ and b, respectively) over the molecule, as shown in Fig. 1e. Fig. 1f shows the measurement scheme and an example of the obtained data and their magnifications. The blue, orange, green, and red lines indicate $X$ - and $Y$-scans, the $Z$-modulation, and the normalized conductance $G / G_{0}$, respectively ( $G_{0}=2 e^{2} / h, h$ is Planck's constant). By plotting the value of $G / G_{0}$ obtained at the STM tip position $(X, Y, Z)$, a 3D volume plot can be obtained, as shown in Fig. $2 \mathrm{a}$ and $\mathrm{b}$. This shows the change in conductance with the conformational change of the single molecule junction. The effect of the sample gradient was removed by linear correction.

\section{Results and discussion}

\section{Experimental results}

Fig. 2a and b show 3D plots obtained for BDA and BDT molecules, respectively. The high-conductance regions in the $X Y$ cross sections have the same periodicity as the $\mathrm{Au}(111)$ atomic structure. Therefore, it is considered that the $\mathrm{Au}_{\mathrm{ads}}$ and the $\mathrm{N}_{\mathrm{S}}$ moved reproducibly on the $\mathrm{Au}(111)$ surface during measurement, which reflects the change in conductance with the position of the $\mathrm{Au}_{\mathrm{ads}}$ and the $\mathrm{N}_{\mathrm{S}}$ on the $\mathrm{Au}(111)$ surface, as was observed in our previous studies. ${ }^{19,20}$ From the high reproducibility of the 3D patterns, the bond structure at the STM tip apex is considered to have been stable during the measurement, showing the single-molecule level analysis reliable.
This measurement method allows the adsorption angle of a molecule ( $\varphi$ in Fig. 2c) to be obtained from the $X Z$ and $Y Z$ cross sections of the conductance map, and the location of the junction point can be obtained from the $X Y$ cross section. ${ }^{19,20}$ Fig. 2c shows a schematic diagram showing the relationship between the $Y Z$ cross section of the conductance map and the adsorption angle of the molecule in the case of BDT. During the conformational change of BDT induced by $Z$-modulation, the location of $\mathrm{Au}_{\mathrm{ads}}$ moves. When $\mathrm{Au}_{\mathrm{ads}}$ is on hollow and ontop sites, the conductance is large and small, respectively. The motion of the molecule induced by $Z$-modulation is schematically shown in Fig. $2 \mathrm{c}$ for the movement of $\mathrm{Au}_{\text {ads }}$ (i) from a hollow site to an on-top site, (ii) from a bridge site to another bridge site via a hollow site, and (iii) from an on-top site to a hollow site.

The conduction depends on the adsorption position of $\mathrm{Au}_{\mathrm{ads}}$ as shown in the color bars in the upper part of each figure in Fig. 2c. When the probe moves in the $X$ - or $Y$-direction, the relationship between the height of the probe and the adsorption position of $\mathrm{Au}_{\text {ads }}$ changes. For example, if the pulling of the molecule starts from a (ii) bridge site and (iii) an on-top site, the probe height when $\mathrm{Au}_{\mathrm{ads}}$ reaches the hollow site changes, resulting in a change in the red squares in the color bars. That is, the $Z$ position at which the conductance becomes large follows that of the upper end of a BDT molecule whose lower end is on a hollow site. Namely, the red line drawn over the color bars corresponds to the trajectory of the upper $\mathrm{Au}_{\mathrm{adt}}$ when an arc of the molecular length is drawn with the lower-end $\mathrm{Au}_{\mathrm{ads}}$ placed on a hollow site, as shown in 
Fig. 2c(iv). Thus, the adsorption angle of the molecule can be derived from the analysis of the arc pattern shown in the $Y Z$ and $X Z$ cross sections, as was carried out in previous studies. ${ }^{19,20}$

Fig. 2d and e show the $Y Z$ cross sections for the cases of $\mathrm{BDA}$ and $\mathrm{BDT}$, respectively. In previous experiments, the results were obtained for a region where the probe-substrate distance was larger than $0.58 \mathrm{~nm}$ for BDA and larger than $0.74 \mathrm{~nm}$ for BDT. Thus, the initial $Z$ distance is shorter in each case in this experiment than in the previous experiments. In the case of BDA, there was no arc-like change in the conductance map (Fig. 2d). This is considered to be due to the BDA molecule not being bound to $\mathrm{Au}_{\mathrm{ads}}$ and $\mathrm{Au}_{\mathrm{adt}}$ and it being almost parallel and closer to the surface in the lying conformation. This allowed conduction pathways to form between the functional groups other than the amino groups, such as benzene rings, and the substrate surface; thus, no site-dependent change in the conductance was observed.

In contrast, in the case of BDT, a bright region was observed in the Z-range of $0.08-0.26 \mathrm{~nm}$, which is considered to be due to the $\mathrm{Au}_{\mathrm{ads}}$ and $\mathrm{Au}_{\mathrm{adt}}$ bound to the end of the BDT, meaning that the number of conductive pathways was limited compared with that in the case of BDA, and a change in the conductance map was observed. However, the $Z$-region with high conductance is wide, which indicates that $\mathrm{Au}_{\mathrm{ads}}$ does not move from a hollow site during the $Z$-modulation of $\sim 0.15 \mathrm{~nm}$ (Fig. 2e). Taking into account the angle of the molecule in the $X Y$ plane and assuming that $\mathrm{BDT}$ is parallel to the substrate at $Z=0$, the angle becomes $20^{\circ}$ and $26^{\circ}$ at $Z=0.1$ and $0.2 \mathrm{~nm}$, respectively (see Fig. $\mathrm{S} 1 \dagger$ and its caption for details).

Fig. 3a and b show two typical conductance curves, namely, the normalized conductance $G / G_{0}$ as a function of the probesubstrate distance for retract and approach motion, obtained for a BDA molecule at positions A and B in Fig. 3c(i). Unlike the results in the previous paper, ${ }^{19}$ where the distance between the probe and the substrate was sufficiently large, no hysteresis appeared and the conductance curves obtained for the retract and approach motion were almost the same. According to the previous results, the hysteresis of the conductance in BDA was found to be due to the difference in the timing of the formation of the bond between the lone pair and the on-top site in the retract and approach motion. Therefore, when the probe-substrate distance was short, as in this case, such a change in the timing did not occur while $Z$ was changed in this range.

The difference between the conductance curve in Fig. 3a and $\mathrm{b}$ is the change observed from (IV) to (V); the conductance became large and then gradually decreased as shown in Fig. 3a, while it continued to gradually increase as shown in Fig. 3b. This difference is considered to be because of the change in the bonding state between the lone pair of $\mathrm{N}_{\mathrm{S}}$ and the $\mathrm{Au}_{\mathrm{ads}}$ atom while the BDA molecule being pulled up and down is not large. Namely, instead of the binding site moving between two adjacent on-top sites, it is considered to move from an on-top site to a bridge site, which does not produce hysteresis.

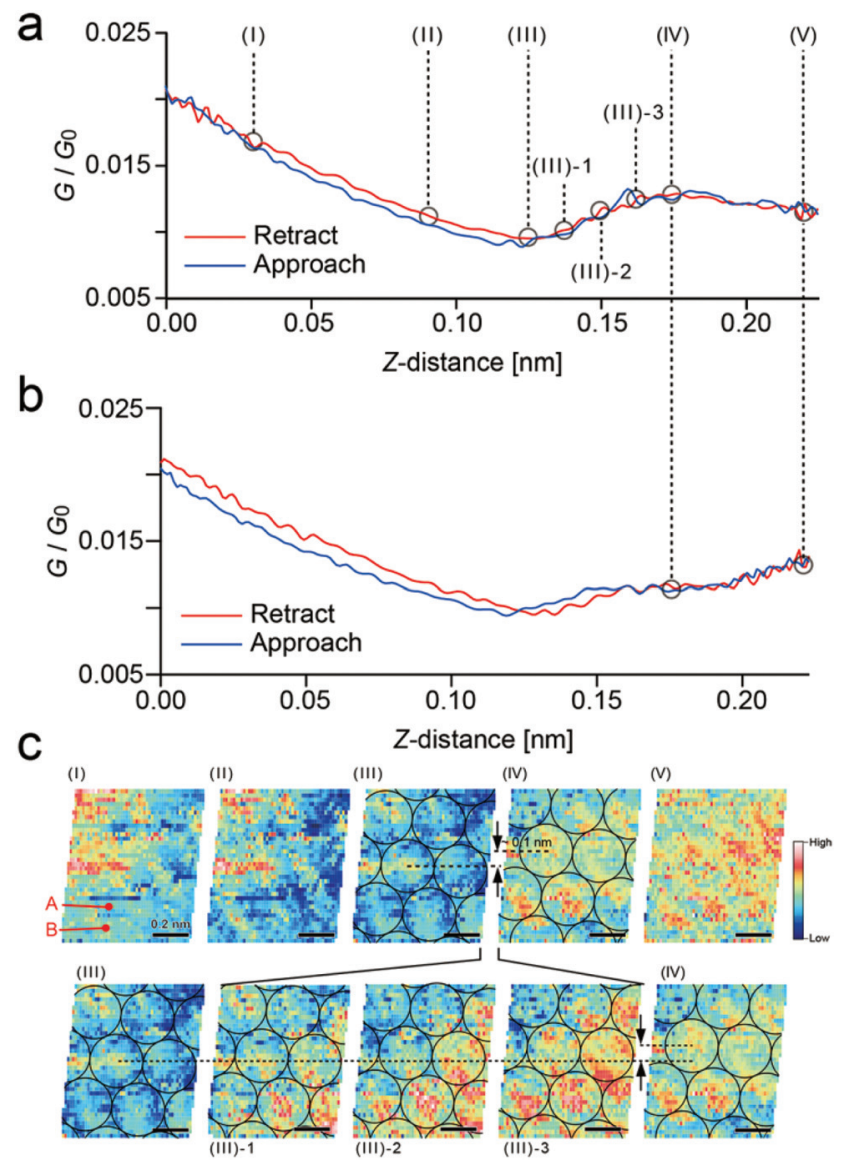

Fig. 3 Data obtained for BDA. (a, b) Two typical conductance curves of a BDA molecule obtained by the retract and approach motion at positions $\mathrm{A}$ and $\mathrm{B}$ in (c) (i). (c) $X Y$ conductance maps corresponding to the $Z$-positions (I) to (V) in (a).

Fig. 3c shows the $X Y$ conductance maps corresponding to the Z-positions (I) to (V) shown in Fig. 3a. The structure of the $\mathrm{Au}(111)$ surface was not observed in Fig. 3c(I), but it started to appear in (III), where the details are shown in the images in the lower row ((III)-1 to (III)-3). This is considered to be due to the fact that BDA stood from the surface and the functional group forming the transmission pathway with the on-top site became only the amino group on the surface side. The atomic pattern of Au was shifted upward by about $0.1 \mathrm{~nm}$ in the $Y$ direction, as indicated by the broken line in Fig. 3c(III) and (IV). Since this is less than the distance between two on-top sites, the shift is considered to be due to the movement of the $\mathrm{Au}_{\mathrm{ad}}$ from an on-top site to a bridge site, as described above.

Fig. $4 \mathrm{a}$ and $\mathrm{b}$ show the conductance curves of BDT and the $X Y$ conductance maps obtained at different probe-substrate distances ((I) to (VIII) in Fig. 4a), respectively. The location where the conductance became large in Fig. 4b was determined in a previous work to be the hollow site on the $\mathrm{Au}(111)$ surface. ${ }^{20}$ As shown in Fig. $4 \mathrm{a}$, similar to the case of BDA, the conductance curves in the retract and approach motion did not show any hysteresis and were almost identical, unlike the 


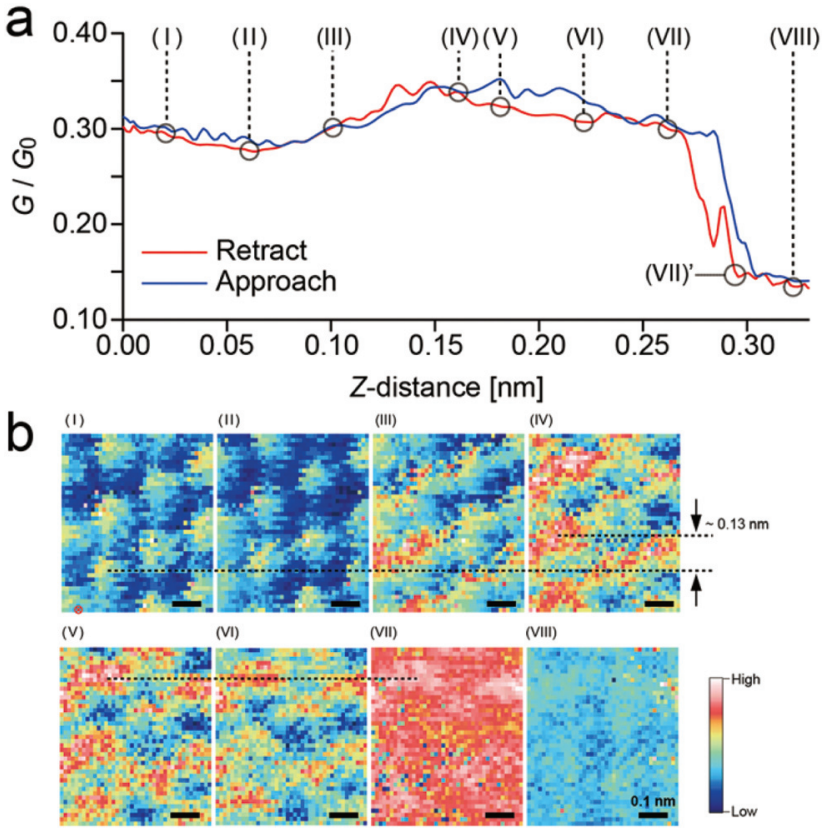

Fig. 4 Data obtained for BDT. (a) Typical conductance curves of a BDT molecule obtained by the retract and approach motion. (b) $X Y$ conductance map corresponding to the Z-positions (I) to (VIII) in (a).

results in the previous work in which the probe-substrate distance was sufficiently large. ${ }^{20}$ Although there is a $\mathrm{Au}_{\mathrm{ads}}$ in the case of BDT, when the distance between the probe and the substrate is short, it is considered that the BDT molecule and $\mathrm{Au}_{\mathrm{ads}}$ do not move significantly on the surface. Namely, this change is considered to be due to the fact that $\mathrm{Au}_{\mathrm{ads}}$ moved between a bridge site and a hollow site via the same pathway for the retract and approach motion instead of between two adjacent hollow sites.

As shown in Fig. 4 b, the conductance map closely reflects the atomic structure of the $\mathrm{Au}(111)$ surface, in contrast to the case of BDA. From (I) to (IV), the contrast gradually changed and, as shown by the broken line in Fig. $4 \mathrm{~b}$, the location where the conductance was large was shifted by about $0.13 \mathrm{~nm}$ (half of the $\mathrm{Au}$ atomic radius), which supports the hypothesis that $\mathrm{Au}_{\mathrm{ads}}$ moved between a bridge site and a hollow site via the same pathway for the retract and approach motion. From (IV) to (VII), $G / G_{0}$ gradually decreased, and although the contrast changed, the location with high conductance did not show any shifts in Fig. 4 b. Then $G / G_{0}$ rapidly decreased from $\sim 0.30$ to $\sim 0.15$ from (VII) to (VII)'. Since the conductance was rapidly halved, it is considered that the number of transmission pathways was halved.

\section{Simulations}

To understand the experimental results in detail, we carried out simulations for BDA and BDT molecules. We used Atomistix ToolKit software (version 12.8.2 Quantum Wise A/S), with DFT within the local density approximation (LDA) using the Perdew-Zunger (PZ) exchange-correlation function ${ }^{27}$ to simulate the optimized structures of the junction and the variation in its conductance while the STM tip was retracted. A double- $\zeta$ basis set was used for all atomic species and the cutoff energy was set to $100 \mathrm{Ry}$. The sampling for Brillouin zone integration was performed at $3 \times 3 \times 400 k$-points. The conductance was calculated by the non-equilibrium Green's function (NEGF) method. ${ }^{28,29} \mathrm{~A}$ bias voltage of $10 \mathrm{mV}$ was applied to the electrodes, and three layers of $\mathrm{Au}(111)$ were prepared. Although DFT underestimates the energy gap, which may result in a larger conductance, the relative change in conductance is reliable. . $^{12,30,31}$

$\mathrm{A} \mathrm{Au}$ atom (adatom) was added to a hollow site on the $\mathrm{Au}(111)$ surface on the upper electrode as the STM tip apex. Structural optimization was first carried out only with a molecule, where BDA was in the cis conformation. The molecular surface was made parallel to the substrate and, as shown in Fig. 1a, it was placed so that both ends were on on-top sites. This condition was used for the calculation for comparison with the experimental result shown in Fig. 3a. Next, when the electrode on the probe side was brought closer, coupling occurred at a certain distance. The height of the upper-electrode-side $\mathrm{N}$ atom from the $\mathrm{Au}(111)$ substrate surface (lower side) was $0.21 \mathrm{~nm}$ lower than the initial height in the previous work $(0.58 \mathrm{~nm}) \cdot{ }^{19}$ First, optimization was performed in this state once. Then the molecule was pulled up step by step (0.3 nm for each step) and optimization was carried out again between each step, where the local pathway and $G$ were obtained. In the calculation for comparison with the experimental result shown in Fig. 3b, the molecule was moved along the $<110>$ direction as the $\mathrm{N}_{\mathrm{S}}$ atom was located on a bridge site. Then a similar procedure to that described above was carried out.

For the BDT molecule, similar optimizations were carried out but with the $\mathrm{Au}$ adatoms $\mathrm{Au}_{\mathrm{adt}}$ and $\mathrm{Au}_{\mathrm{ads}}$ at both ends of the molecule, which were placed at hollow sites in accordance with the structure shown in Fig. 1b. After the first structural optimization and the calculation of the conductance, the distance between the two electrodes was changed in steps of $0.02 \mathrm{~nm}$ up to $0.34 \mathrm{~nm}$. The upper-electrode-side Au adatom $\left(\mathrm{Au}_{\mathrm{adt}}\right)$ was fixed during the whole process to reflect the stable junction structure at the STM tip apex because highly reproducible conductance patterns were obtained in measurements. Since more detailed analysis is necessary for the calculations while the STM tip is made to approach the substrate, simulations were carried out for the case of STM tip retraction here.

First we discuss the case of BDA. Fig. 5a shows a conductance curve obtained for BTA, which is in good agreement with the experimental result shown in Fig. 3a. Fig. 5b shows the conformations and local transmission contributions obtained for (i) to (v) in Fig. 5a, which correspond to the change from (I) to (V) in Fig. 3a. Structural optimization was first carried out only with a free molecule, where BDA has a cis conformation. The molecular surface was set parallel to the substrate and it was placed so that both ends were on the on-top sites as shown in Fig. 1a. Then, as the electrode on the probe side was brought closer, coupling occurred at a certain distance. 
a

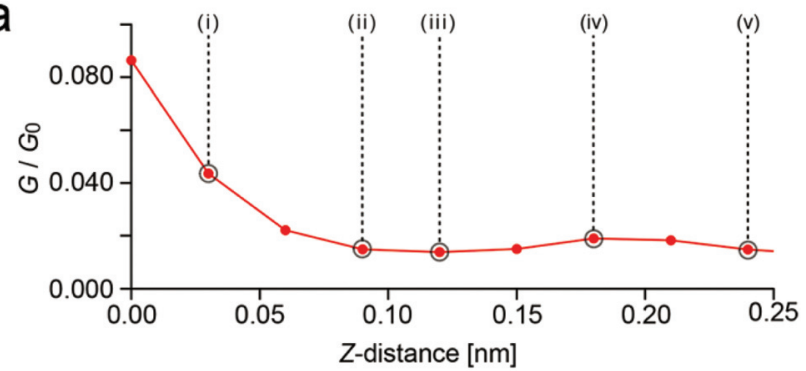

b

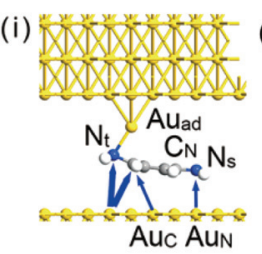

(iv)
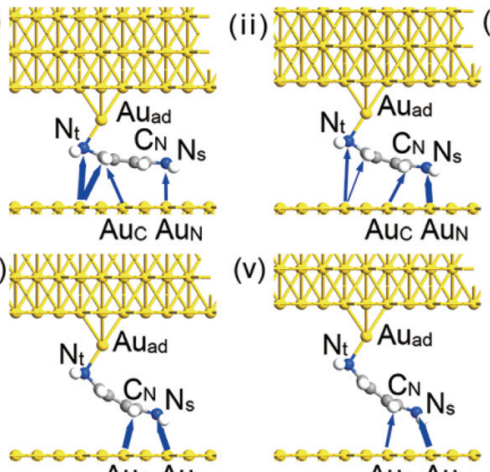

(iii) DXNXNXNXND
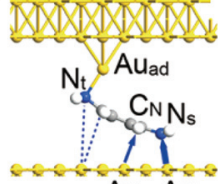

(v)

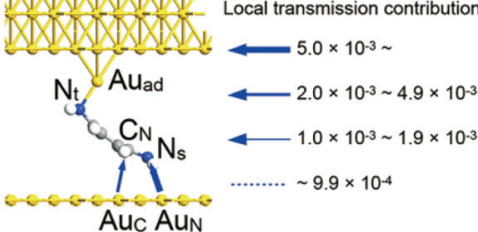

Fig. 5 Simulated conductance curve obtained for BDA. (a) Simulated conductance curve corresponding to the change from (I) to (V) in Fig. 3a. (b) Schematics of conformational structures and local transmission contributions for (i) to (v) in (a). Local transmission contributions are proportional to the thickness of the arrows.

Optimization was first performed in this state. Then the molecule was pulled up step by step ( $0.03 \mathrm{~nm}$ for each step) and optimization was carried out between each step, where the local pathway and $G$ were obtained. Fig. $5 \mathrm{~b}(\mathrm{i})$ shows the optimized conformation after one step.

In contrast to the previous work,${ }^{19}$ the site of the bond did not change significantly while the distance between two electrodes was short. In (i), several transmission pathways were formed around the functional groups other than the amines of $\mathrm{BDA}$, such as the benzene ring and current flowed from some other sites on the substrate, rather than an electron flow through the benzene ring of BDA. The existence of several local transmission pathways is considered to be the reason for the low contrast of the atomic structures shown in Fig. 3c. In (iii), the contribution of $\mathrm{Au} / \mathrm{N}_{\mathrm{t}} / \mathrm{Au}$ to the local transmission decreases and the contribution of the local transmission near the Ns atom becomes large, which is considered to have made the atomic structure in Fig. $3 \mathrm{c}$ clearer.

In Fig. 5b(i)-(iii), the position of the Ns atom slightly moves from the on-top site, strengthening the transmission pathway formed by the coordination bond ${ }^{15}$ between the lone pair of $\mathrm{N}_{\mathrm{S}}$ and $\mathrm{Au}_{\mathrm{N}}$. However, as the distance between $\mathrm{N}_{\mathrm{t}}$ and the substrate increases, its transmission decreases, resulting in the observed decrease in $G / G_{0}$ from (i) to (iii). In (iii), $\mathrm{N}_{\mathrm{S}}$ is above $\mathrm{Au}_{\mathrm{N}}$ and forms a transmission pathway via the coordination bond ${ }^{19}$ between the lone pair of $\mathrm{N}_{\mathrm{S}}$ and $\mathrm{Au}_{\mathrm{N}}$. At this time, since $\mathrm{C}_{\mathrm{N}}$ is not above $\mathrm{Au}_{\mathrm{C}}$, its contribution is small, and the conductance between $\mathrm{N}_{\mathrm{S}}$ and $\mathrm{Au}_{\mathrm{N}}$ can be observed in the conductance map. In (iv), since $\mathrm{C}_{\mathrm{N}}$ moves above $\mathrm{Au}_{\mathrm{C}}$, forming two transmission pathways of $\mathrm{N}_{\mathrm{S}}-\mathrm{Au}_{\mathrm{N}}$ and $\mathrm{C}_{\mathrm{N}}-\mathrm{Au}_{\mathrm{C}}$, the conductance becomes large. In $(\mathrm{v}), \mathrm{C}_{\mathrm{N}}$ is pulled away from the substrate and the contribution of the conduction between $\mathrm{C}_{\mathrm{N}}$ and $\mathrm{Au}_{\mathrm{C}}$ to the transmission becomes small. These changes closely reproduce the observed result. From the positions of the $\mathrm{N}_{\mathrm{S}}$ atom between Fig. $5 \mathrm{~b}$ (iii) and (iv), the displacement of the $\mathrm{N}_{\mathrm{S}}$ atom was estimated to be $0.11 \mathrm{~nm}$, which is in agreement with the displacement in the conductance map in Fig. 3c $(0.1 \mathrm{~nm})$.

To reproduce the conductance curve in Fig. 3b, the initial structure was changed and calculations were carried out. The initial position of the molecular end was moved away from the on-top site. Namely, the bond structure between $\mathrm{Au}_{\mathrm{ad}}$ and $\mathrm{N}_{\mathrm{t}}$, and the position of $\mathrm{N}_{\mathrm{S}}$ were changed.

Although calculations were carried out for various distances moved, almost the same results were obtained. Fig. $6 \mathrm{a}$ and $\mathrm{b}$ show the calculated conductance curve and the schematic structures corresponding to (i) to (v) in Fig. 6a, respectively. The conductance curve was in good agreement with the experimental curve shown in Fig. 3b.

The positional relationship between $\mathrm{Au}_{\mathrm{ad}}$ and $\mathrm{N}_{\mathrm{t}}$ was fixed similarly to that in a previous work ${ }^{20}$ so that the relative position between the upper electrode as the probe and $\mathrm{N}_{\mathrm{t}}$ did not change while raising the BDA molecule. The calculated contributions of the local transmission ${ }^{22-24}$ between ${A u_{a d}}_{\text {and }} \mathrm{N}_{\mathrm{t}}$

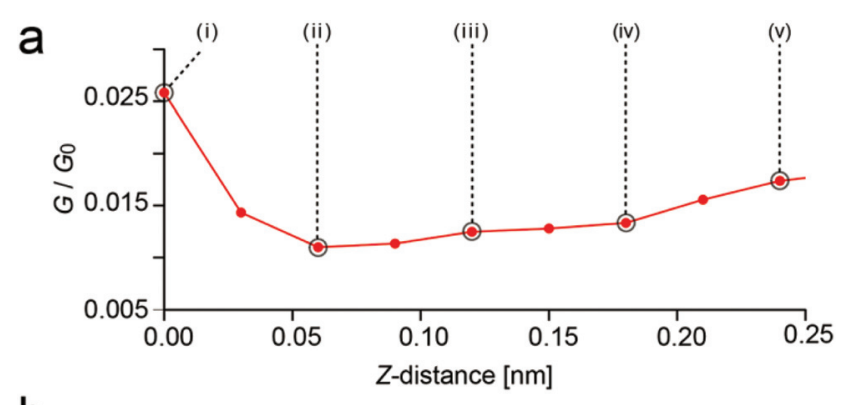

$b_{(i)}$
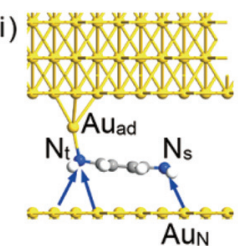

(iv)
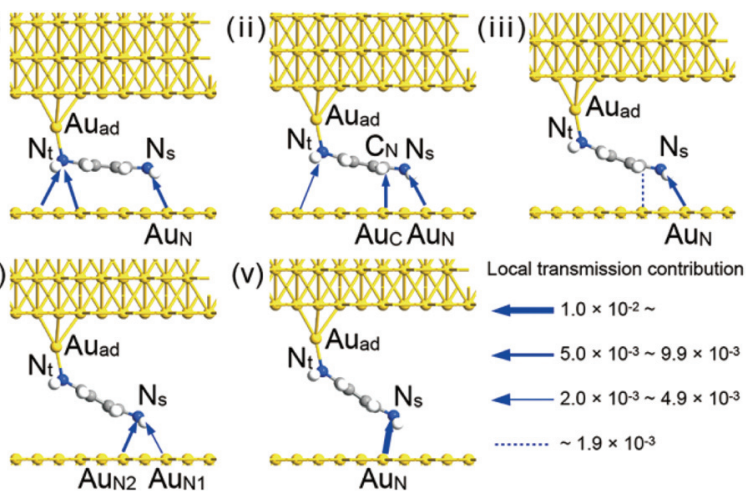

Fig. 6 Simulated conductance curve obtained for BDA. (a) Simulated conductance curve corresponding to the change from (I) to (V) in Fig. 4b. (b) Schematics of conformational structures and local transmission contributions for (i) to (v) in (a). Local transmission contributions are proportional to the thickness of the arrows. 
shown in Fig. $6 \mathrm{~b}$ gradually decreased from (iii) to (v) $\left(3.1 \times 10^{-2}\right.$, $2.7 \times 10^{-2}$, and $2.4 \times 10^{-2}$, respectively), differing from the observed change shown in Fig. 6a, which is due to the increase in the angle between $\mathrm{N}_{t}$ and the BDA molecular plane. In addition, although $\mathrm{N}_{\mathrm{S}}$ moves from an on-top site to a bridge site from (i) to (v) in Fig. $5 \mathrm{~b}, \mathrm{~N}_{\mathrm{S}}$ is at a bridge site with low conductivity for (i) to (iii) and moves to an on-top site with high conductivity in Fig. 6b. Actually, the calculated contribution of the local transmission between $\mathrm{N}_{\mathrm{S}}$ and $\mathrm{Au}_{\mathrm{N}}$ increased from (i) to (iii) $\left(7.9 \times 10^{-3}, 8.4 \times 10^{-3}\right.$, and $1.2 \times 10^{-2}$, respectively), resulting in an increase in the total conductance, which is in good agreement with the observed change from (iii) to (v) shown in Fig. 6a.

Although it was difficult to directly evaluate the adsorption energy because of the existence of the STM tip which pulled up the molecule, the interaction between the BDA lone pair and $\mathrm{Au}$ atom was dominant even in the lying form in (i) and (ii). The binding between $\mathrm{C}_{\mathrm{N}}$ and $\mathrm{Au}_{\mathrm{C}}$ was weaker than that via the lone pair. As the conformation changed into the standing form as (iii) to (v), the binding became to be a single interaction. The structure maintained for a further pulling position as shown in a previous paper, ${ }^{19}$ which is in good agreement with the idea that relatively sharp conductance histogram peaks were observed for the amine-Au system due to the relatively weak gold-amine interaction, namely, amines can only significantly bind to gold adatoms in a standing form. ${ }^{12}$

As has been shown, the dynamics of the bond state in the BDA single-molecule junction for a small probe-substrate distance was well explained by the results obtained by combining the 3D dynamic probe method and DFT calculations. A similar simulation was then carried out for the BDT molecule. Fig. 7a shows the conductance curve obtained by the simulation. The change in conductance from (i) to (iv) is in good agreement with the observed change shown in Fig. 4a. However, the calculated conductance rapidly decreases between (iv) and (vi) and gradually decreases from (vi) to (viii), while in the experiments, it rapidly decreased after a gradual decay.

Fig. $7 \mathrm{~b}$ shows the conformations used for the calculations and the obtained contributions to the local transmission. Similar simulation procedures to those discussed earlier were carried out (see Experimental for the initial conditions). From (i) to (iv), because the contributions to the local transmission via the $\mathrm{Au}_{\text {adt }}-\mathrm{S}_{\mathrm{t}}$-substrate (upper side) and $\mathrm{Au}_{\text {ads }}-\mathrm{S}_{\mathrm{S}}$-substrate (lower side) are larger than that via the phenyl group (benzene ring) of the BDT transmission pathway, there is not a single transmission pathway passing through the BDT molecule, and these are two dominant pathways at both ends of the molecule shown by the pink arrows.

In the change from (iv) to (vi), the local transmission contributions of these two pathways become smaller and one of them disappears. The calculated values of $G / G_{0}$ for (iv) and (v)' were 1.11 and 0.53 , respectively, in good agreement with the decrease to the values shown in Fig. 4a (VII) and (VII)'. This rapid change in conductance is due to the rotation of the benzene ring of BDT as shown in Fig. 7. Then the local transmission contribution via the phenyl group (benzene ring)
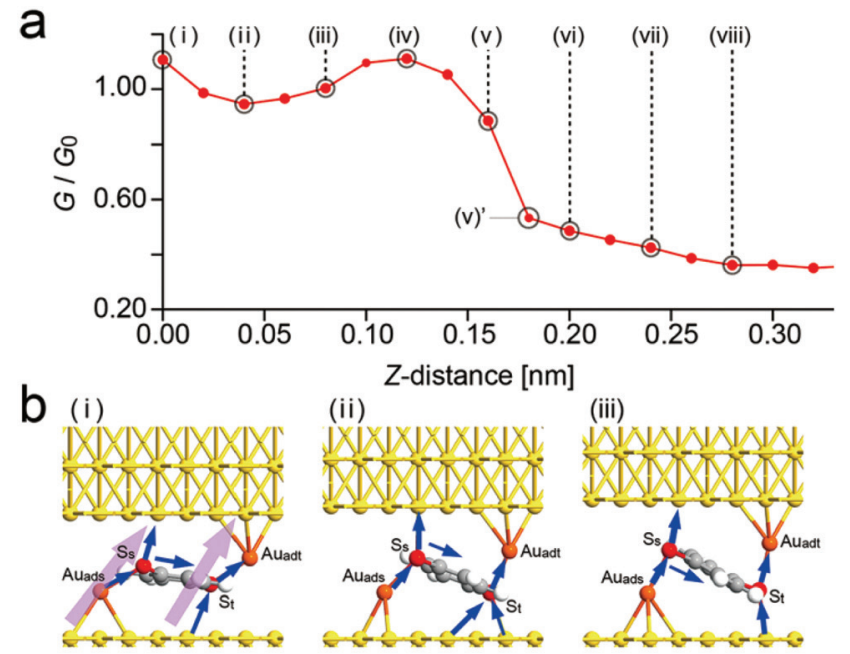

(ii)

Z-distance [nm]

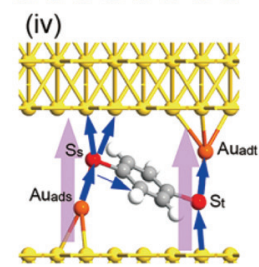

(vii)
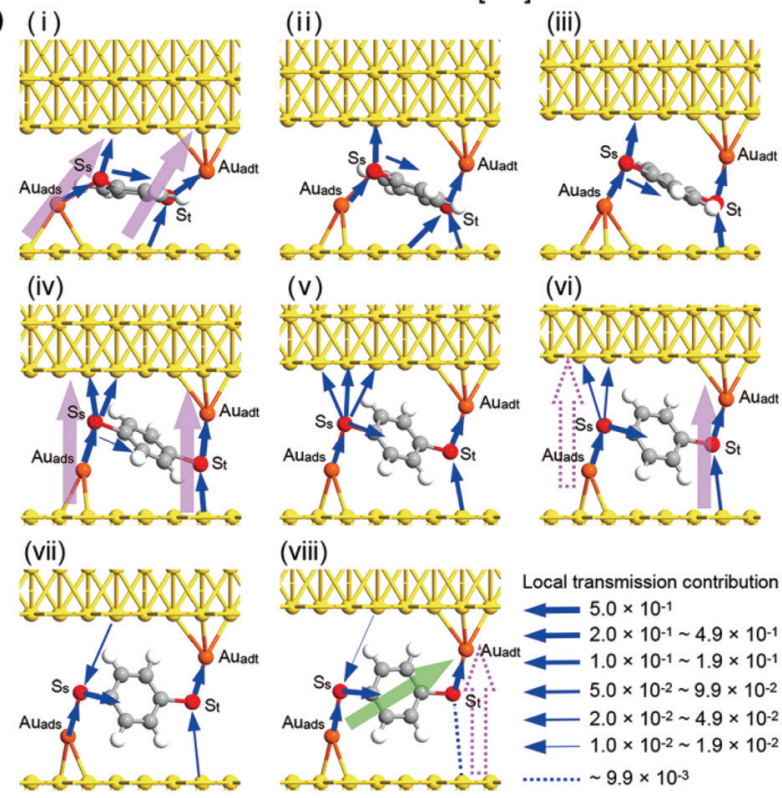

(viii)

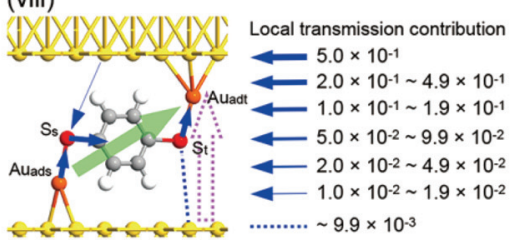

Fig. 7 Simulated conductance curve obtained for BDT. (a) Simulated conductance curve corresponding to the change in Fig. 4a. (b) Schematics of conformational structures and local transmission contributions for (i) to (viii) in (a). The direction of the arrows from the upper substrate to $S_{S}$ shown in (vii) and (viii) indicates the negative contribution. Local transmission contributions are proportional to the thickness of the arrows.

becomes dominant, as shown by the light green arrow in (viii), which decreases with the increase in $Z$, producing the gradual decrease in the conductance shown in Fig. 7a.

In the experiment, a gradual decrease in conductance was observed from (IV) to (VII) before the rapid change from (VII) to (VIII) as shown in Fig. 4a. This is considered to be because the rotation of BDT begins after the two $\mathrm{Au} / \mathrm{S} / \mathrm{Au}$ pathways become one in the simulation, while the rotation occurred first in the experiment. This difference is considered to be due to the actual tip surface not being flat similarly to the substrate used for the calculation, but having a pyramidal structure, which reduces the effect of steric hindrance in the calculation.

To see the conformational change in the molecule in more detail, Fig. 8 shows the conformations shown in Fig. $7 \mathrm{~b}$ as seen from the $Z$-direction. $\mathrm{Au}_{\mathrm{ads}}$ moves from a hollow site to a bridge site. The distance moved is $0.15 \mathrm{~nm}$, as shown by the broken line, which is in good agreement with the value of $0.13 \mathrm{~nm}$ experimentally obtained for the change from (I) to 

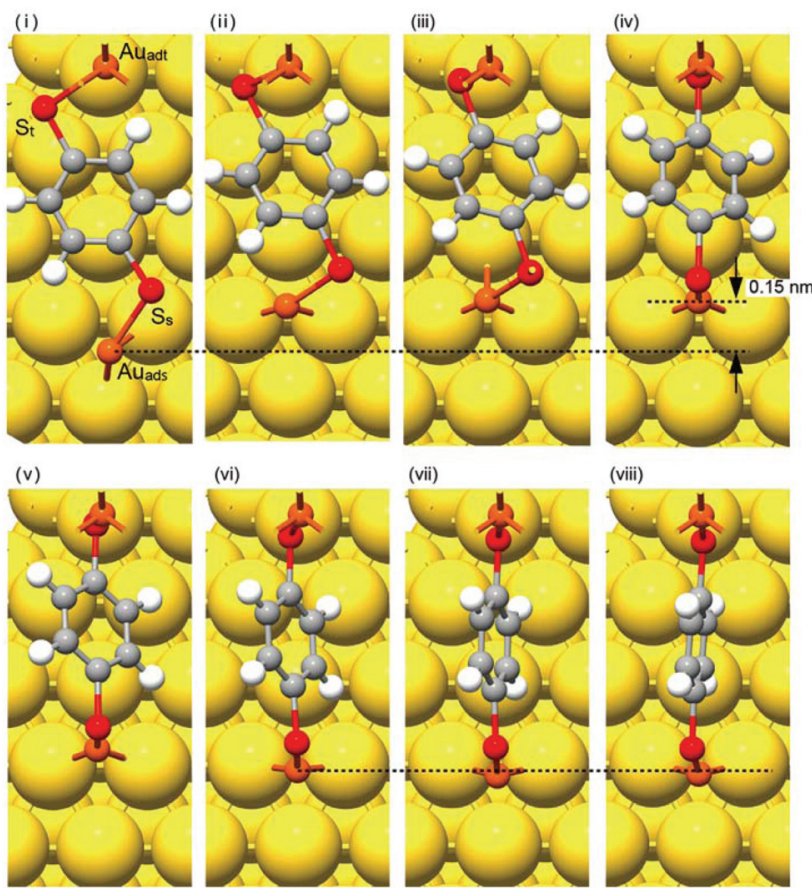

Fig. 8 Schematic structures of BDA on a $A u(111)$ surface. Conformations of BDA shown in Fig. 7b as seen from the $Z$-direction.

(IV) as shown in Fig. 4b. $\mathrm{Au}_{\mathrm{ads}}$ does not move from (vi) to (viii) while the molecule is pulled up, as shown by the broken line, which is considered to be due to the rotation of the molecule during the change in $Z$.

\section{Conclusions}

By performing analysis on 1,4-benzenediamine (BDA) and 1,4-benzenedithiol (BDT) single molecule junctions by the 3D dynamic probe method and DFT calculations, we have observed, for the first time, the effect of a change in the molecular conformations and bonding states on the local transmission pathways for a short $\mathrm{Au}$ electrode distance. The obtained results are expected to play an important role in advancing the development of nanoscale devices making full use of the characteristics of single molecules.

\section{Conflicts of interest}

There are no conflicts to declare.

\section{Acknowledgements}

H.S. and S.Y. acknowledge the financial support from the Japan Society for the Promotion of Science, the Grant-in-Aid for Scientific Research 17H06088, and the Grant-in-Aid for Young Scientists (A) 16H05982, respectively.

\section{Notes and references}

1 D. Kahanda, K. T. DuPrez, E. Hilario, M. A. McWilliams, C. H. Wohlgamuth, L. Fan and J. D. Slinker, Anal. Chem., 2018, 90, 2178-2185.

2 Y. van de Burgt, E. Lubberman, E. J. Fuller, S. T. Keene, G. C. Faria, S. Agarwal, M. J. Marinella, A. Alec Talin and A. Salleo, Nat. Mater., 2017, 16, 414.

3 C. Santoro, C. Arbizzani, B. Erable and I. Ieropoulos, J. Power Sources, 2017, 356, 225-244.

4 A. J. Bandodkar, C. S. López, A. M. Vinu Mohan, L. Yin, R. Kumar and J. Wang, Sci. Adv., 2016, 2, e1601465.

5 S. V. Aradhya and L. Venkataraman, Nat. Nanotechnol., 2013, 8, 399.

6 C. Bruot, J. Hihath and N. Tao, Nat. Nanotechnol., 2011, 7, 35.

7 M. S. Hybertsen and L. Venkataraman, Acc. Chem. Res., 2016, 49, 452-460.

8 S. Kaneko, D. Murai, S. Marqués-González, H. Nakamura, Y. Komoto, S. Fujii, T. Nishino, K. Ikeda, K. Tsukagoshi and M. Kiguchi, J. Am. Chem. Soc., 2016, 138, 12941300.

9 M. Kiguchi, H. Nakamura, Y. Takahashi, T. Takahashi and T. Ohto, J. Phys. Chem. C, 2010, 114, 22254-22261.

10 Y. Komoto, S. Fujii, H. Nakamura, T. Tada, T. Nishino and M. Kiguchi, Sci. Rep., 2016, 6, 26606.

11 S. Y. Quek, M. Kamenetska, M. L. Steigerwald, H. J. Choi, S. G. Louie, M. S. Hybertsen, J. B. Neaton and L. Venkataraman, Nat. Nanotechnol., 2009, 4, 230.

12 S. Y. Quek, L. Venkataraman, H. J. Choi, S. G. Louie, M. S. Hybertsen and J. B. Neaton, Nano Lett., 2007, 7, 34773482.

13 H. Vazquez, R. Skouta, S. Schneebeli, M. Kamenetska, R. Breslow, L. Venkataraman and M. S. Hybertsen, Nat. Nanotechnol., 2012, 7, 663.

14 L. Venkataraman, J. E. Klare, C. Nuckolls, M. S. Hybertsen and M. L. Steigerwald, Nature, 2006, 442, 904.

15 L. Venkataraman, J. E. Klare, I. W. Tam, C. Nuckolls, M. S. Hybertsen and M. L. Steigerwald, Nano Lett., 2006, 6, 458-462.

16 B. Xu and N. J. Tao, Science, 2003, 301, 1221-1223.

17 Y. Kitaguchi, S. Habuka, H. Okuyama, S. Hatta, T. Aruga, T. Frederiksen, M. Paulsson and H. Ueba, Sci. Rep., 2015, 5, 11796.

18 M. Nakamura, S. Yoshida, T. Katayama, A. Taninaka, Y. Mera, S. Okada, O. Takeuchi and H. Shigekawa, Nat. Commun., 2015, 6, 8465.

19 Y. Sugita, A. Taninaka, S. Yoshida, O. Takeuchi and H. Shigekawa, Sci. Rep., 2018, 8, 5222.

20 S. Yoshida, A. Taninaka, Y. Sugita, T. Katayama, O. Takeuchi and H. Shigekawa, ACS Nano, 2016, 10, 1121111218.

21 S. Yasuda, S. Yoshida, J. Sasaki, Y. Okutsu, T. Nakamura, A. Taninaka, O. Takeuchi and H. Shigekawa, J. Am. Chem. Soc., 2006, 128, 7746-7747. 
22 C. M. Guédon, H. Valkenier, T. Markussen, K. S. Thygesen, J. C. Hummelen and S. J. van der Molen, Nat. Nanotechnol., 2012, 7, 305.

23 G. C. Solomon, C. Herrmann, T. Hansen, V. Mujica and M. A. Ratner, Nat. Chem., 2010, 2, 223.

24 X. Xu, W. Li, X. Zhou, Q. Wang, J. Feng, W. Q. Tian and Y. Jiang, Phys. Chem. Chem. Phys., 2016, 18, 3765-3771.

25 J. Kestell, R. Abuflaha, M. Garvey and W. T. Tysoe, J. Phys. Chem. C, 2015, 119, 23042-23051.

26 Z. Li and D. S. Kosov, Phys. Rev. B: Condens. Matter Mater. Phys., 2007, 76, 035415.
27 J. P. Perdew and A. Zunger, Phys. Rev. B: Condens. Matter Mater. Phys., 1981, 23, 5048-5079.

28 M. Brandbyge, J.-L. Mozos, P. Ordejón, J. Taylor and K. Stokbro, Phys. Rev. B: Condens. Matter Mater. Phys., 2002, 65, 165401.

29 J. Taylor, H. Guo and J. Wang, Phys. Rev. B: Condens. Matter Mater. Phys., 2001, 63, 245407.

30 F. Flores, J. Ortega and H. Vázquez, Phys. Chem. Chem. Phys., 2009, 11, 8658-8675.

31 J. C. Cuevas and E. Scheer, Molecular Electronics: An Introduction to Theory and Experiment, World Scientific, 2010. 